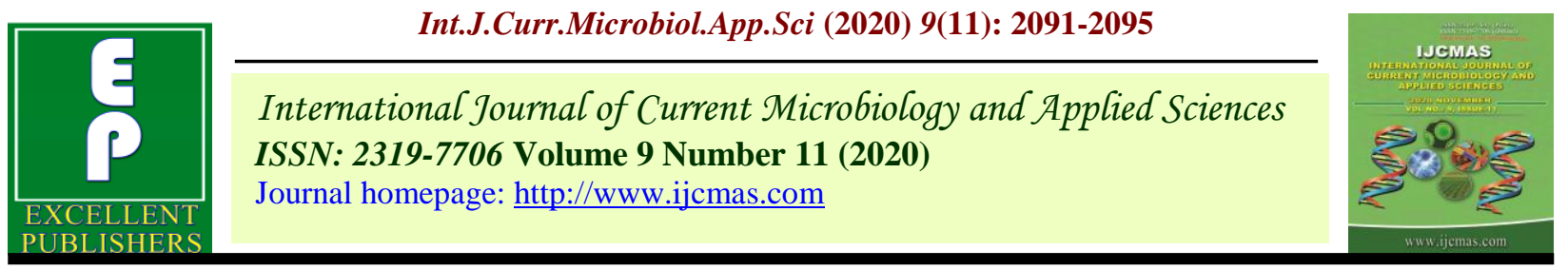

Original Research Article

https://doi.org/10.20546/ijcmas.2020.911.249

\title{
Chondroprotective Effect of Withania somnifera in Broilers Treated with High Doses of Enrofloxacin
}

\author{
A. Arivuchelvan ${ }^{1 *}$ and S. Murugesan ${ }^{2}$ \\ ${ }^{1}$ Department of Veterinary Pharmacology and Toxicology, Veterinary College and Research \\ Institute, Namakkal -637 002, Tamil Nadu, India \\ ${ }^{2}$ Veterinary College and Research Institute, Theni, Tamil Nadu, India \\ *Corresponding author
}

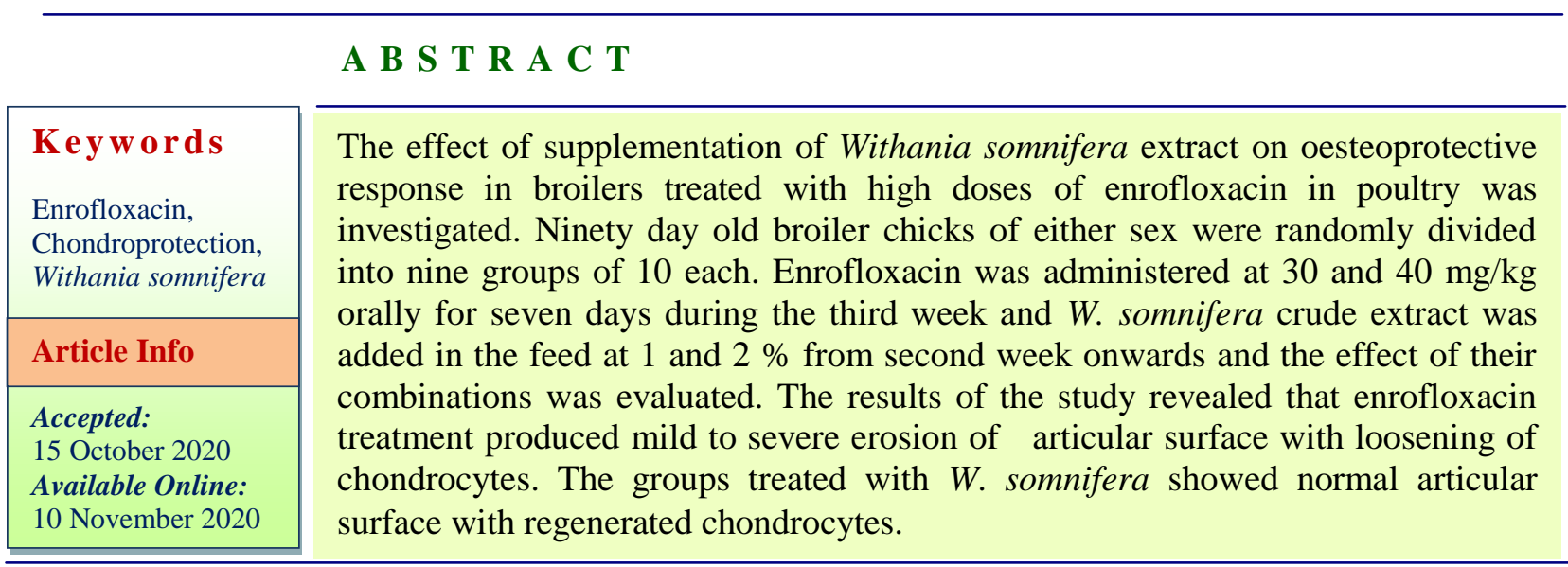

\section{Introduction}

Antibacterials especially enrofloxacin is used frequently by poultry farmers at very high doses from the first week to culling/marketing. The impact analysis of this practice revealed many negative effects namely failure of treatment, escalation of treatment cost, adverse effects on mammalian cells and development of resistance. Withania somnifera is an herb that grows naturally in diverse areas ranging from Africa, the Mediterranean East and India. It has earned the nickname "Indian Ginseng". The chemistry of Withania somnifera has been extensively studied and over 35 chemical constituents have been identified. Discarnate use of antibiotics, is a common feature encounted in poultry farms especially in Tamil Nadu. Administration of antibacterials without proper recommendation and supervision is not uncommon. Self prescription or prescription from unqualified input dealers are increasing, causing major problem not only to the farms and farmers but also to the end users of poultry products.

Gentamicin and Enrofloxacin are the two handy drugs among the antibacterials, which are used frequently by poultry farmers at very 
high doses from the day of hatching toculling/marketing. The impact analysis of this practice reveals many negative effects namely failure of treatment, adverse reaction of antibacterials and escalation of treatment cost. The biologically active constituents are with anolides $\mathrm{G}$ and $\mathrm{D}$, withaferin $\mathrm{A}$, isopelletierine, anaferine, with asomnine etc. (Mishra et al., 2000). These are believed to be responsible for its diverse pharmacological actions viz., anticonvulsant, antiinflammatory, antioxidant, antitumor, anxiolytic actions (Bhattacharya et al., 2001). Hence the present study was planned to explore the possible chondroprotective effect of Withania somnifera in broilers treated with high doses of enrofloxacin.

\section{Materials and Methods}

Commercial day old, unsexed broiler straight run chicks (Vencob strain) belonging to a single hatch, obtained from a commercial hatchery at Namakkal were used for the experimental study. All the chicks were reared under standard and uniform managemental conditions throughout the experimental period of six weeks. Broiler starter and finisher mash, free of toxins and pesticide residues purchased from a feed manufacturing unit at Veterinary College and Research Institute, Namakkal was used as a basal diet for formulating the experimental diet. The broiler starter and finisher mashes were fed ad libitum to the birds from 1 to 28 and 29 to 42 days of age respectively.

The crude extracts of the herbal plant Withania somnifera (root) obtained from Natural Remedies, Bangalore and commercially available enrofloxacin (Enrocin) was used for the experimental study. A survey was conducted to fix the dose of enrofloxacin. Inclusion levels of plant extract were fixed as per the literature.

Experimental diets containing W. somnifera root extract (crude) were prepared and mixed with feed and fed to the following treatment groups. On first day, all ninety broiler chicks were weighed with banded wing and reared in battery brooders. On eighth day, the chicks were randomly divided into nine treatment groups of ten each.

Commercial enrofloxacin was given during third week for seven days (i.e., 15th to 21 st day) through drinking water as pulsed dosing. The birds were subjected to respective treatment from eighth day to forty second day at various dose levels of the drug and different inclusion levels of the plant as per table 1.

\begin{tabular}{|c|c|l|}
\hline S. No. & Treatment & Experimental group \\
\hline $\mathbf{1}$ & T1 & Normal control \\
\hline $\mathbf{2}$ & T2 & Enrofloxacin- 30mg/kg \\
\hline $\mathbf{3}$ & T3 & Enrofloxacin- 40mg/kg \\
\hline $\mathbf{4}$ & T4 & Withania somnifera- $1 \%$ level \\
\hline $\mathbf{5}$ & T5 & Withania somnifera- $2 \%$ level \\
\hline $\mathbf{6}$ & T6 & Enrofloxacin $-30 \mathrm{mg} / \mathrm{kg}+$ Withania somnifera $-1 \%$ level \\
\hline $\mathbf{7}$ & T7 & Enrofloxacin $-40 \mathrm{mg} / \mathrm{kg}+$ Withania somnifera $-1 \%$ level \\
\hline $\mathbf{8}$ & T8 & Enrofloxacin $-30 \mathrm{mg} / \mathrm{kg}+$ Withania somnifera $-2 \%$ level \\
\hline $\mathbf{9}$ & T9 & Enrofloxacin $-40 \mathrm{mg} / \mathrm{kg}+$ Withania somnifera $-2 \%$ level \\
\hline
\end{tabular}


The bone samples (femur) were collected in buffered neutral formalin for histopathology and sections were prepared and stained by haematoxylin and eosin.

\section{Results and Discussion}

\section{Histopathology}

The histopathological section of femur at the end of sixth week from normal control group revealed normal uninterrupted articular surface with intact chondrocytes (Plate 1).
The enrofloxacin control group T2 had shown mild erosion of articular surface with loosening of chondrocytes (Plate 2). The group T3 treated with higher dose of enrofloxacin recorded severe erosion of articular surface with destruction of chondrocytes (Plate 3). The treatment group T8 showed normal articular surface with regenerated chondrocytes (Plate 4). Normal articular surface with regenerating chondrocytes was noticed in the treatment group T9 (Plate 5).

Plate.1 Normal Control - Femur showing normal uninterrupted articular surface with intact chondrocytes H \& E 400X

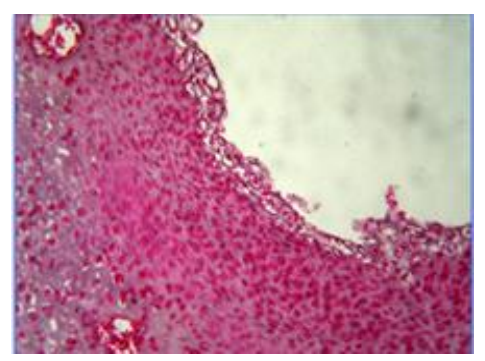

Plate.2 EF (30mg) - Femur showing mild erosion of articular surface with loosening of chondrocytes H \& E 400X

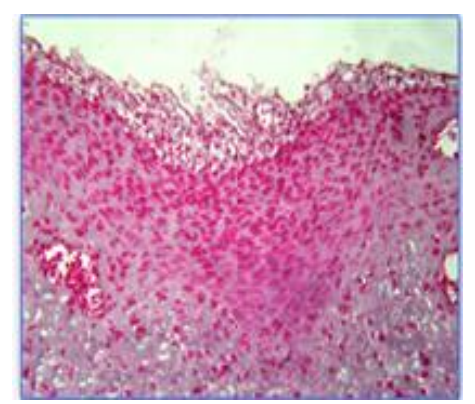

Plate.3 EF (40mg) - Femur showing severe erosion of articular surface with destruction of chondrocytes H \& E 400X

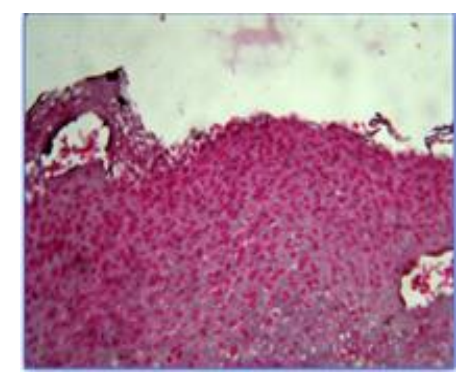



Plate.4 WS (2\%) + EF (30mg) - Femur showing normal articular surface with regenerated
chondrocytes H \& E 400X

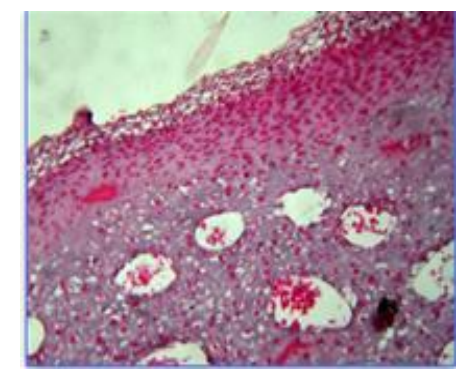

Plate.5 WS (2\%) + EF (40mg) - Femur showing normal articular surface with regenerating chondrocytes H \& E 400X

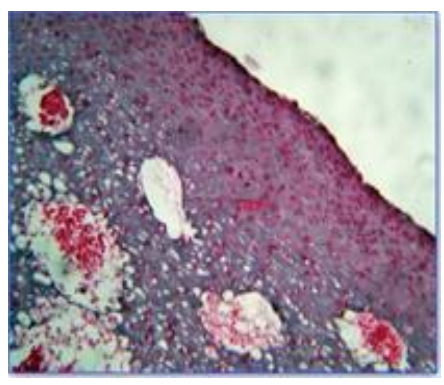

Enrofloxacin and ciprofloxacin, synthetic chemotherapeutic agents belonging to the class of fluroquinolones, are widely used in broilers to control many bacterial infections. (Sarraga et al., (2006); Pulverer et al.,(1986) At high doses, these drugs are reported to cause toxic effects including inhibition of the neurotransmitter GABA, central nervous system excitement, convulsion, ocular problems and arthropathy. Chondrotoxic effects of fluroquinolones have been elaborately studied in human beings. Vitamins and minerals have been tried against quinolones induced chondrotoxicity.

The histopathology of the bone of the control group revealed normal uninterrupted articular surface with intact chondrocytes. The bone of enrofloxacin treated group revealed mild to severe erosion of articular surface with destruction of chondrocytes in a dose dependent manner (Maslanka et al., 2009; Udayakumar et al., 2009) also reported that enrofloxacin at high dose, exceeding the therapeutic level produces damage of articular cartilage damage in chicken. Similar arthropathic changes were reported by (Stalhmann, 1991; peters et al., 2002). The most possible mechanism for the initiation of quinolone induced arthropathy was chelation of calcium and magnesium ions by quinolones which in turn result in alters the functions of chondrocyte surface integrin receptors (Stalhmann et al., 1995). Withania somnifera control group at both the doses revealed normal architecture of articular surfaces and chondrocytes. The treatment groups revealed dose dependent improvement in the regeneration of chondrocytes and articular surfaces. (Rassool and Varalakshmi, 2006) also reported that Withania somnifera root powder reversed the arthropathic changes on experimental gout arthritis both in vivo and in vitro. Withania somnifera has been proved to increases serum calcium and decrease serum phosphorus and alkaline 
phosphatase level and thereby prevented osteoporosis (Irshad Ahmed, 2002).

\section{References}

Bhattacharya, A., S. Ghosal and S. K. Bhattacharya, 2001. Anti-oxidant effect of Withania somifera glycowithanolides in chronic foot shock stress-induced perturbations of oxidative free radical scavenging enzymes and lipid peroxidation in rat frontal cortex and striatum. J. Ethnopharmacol., 74: 1-6.

Irshad Ahmed, 2002. Efficacy of OST-6, a polyherbal formulation in the management of osteoporosis in postmenopausal women. Orthopaedics Today, 4 (IV): 241-244.

Maslanka, T., J. J. Jaroszewski, A. Mikolajczyk and T. Rotkiewicz, 2009. Effect of increasing doses of enrofloxacin on chicken articular cartilage. Pol. J. Vet. Sci., 12(1): 21-33.

Mishra, L. C., B. B. Singh and Simon Dagenais, 2000. Scientific basis of the therapeutic use of Withania somnifera (Ashwagandha): A Rev. Alt. Med. Rev., 5(4): 334-345.

Peters, T. L., R. M. Fulton, K. D. Robertson and M. W. Orth, 2002. Effects of antibiotics on in vitro and in vivo avian cartilage degradation. Avian Dis., 46(1): 75-86.

Pulverer, G., W. Roszkowski, H. L. Ko, K. Roszkowski and J. jeljaszewicz, 1986. Animal experiment studies on the modification of the immune system by ofloxacin. Infection, 14(1): 40-44.

Rassool, $\mathrm{M}$ and P. Varalakshmi, 2006.
Suppressive effect of Withania somnifera root powder on experimental gouty arthritis: An in vivo and in vitro study. World J. Med. Sci., 2(4): 16-24.

Sarraga, C., I. Carreras, J. A.Garcia regueiro and M. Castellari, 2006. The combined effects of alpha tocopheryl acetate supplementation and enrofloxacin administration of oxidative stability of turkey meat. British Poult. Sci., 47(6): 708-713.

Stahlman, R., C. Forster, M. Shakibaei, J. Vormann, T. Gunther and H. J. Merker, 1995. Magnesium deficiency induces joint cartilage lesions in juvenile rats which are identical to quinolone induced arthropathy. Antimicrob. Agents Chemother., 39(9): 2013-2018.

Stahlmann, R., P2010. Safety consideration of fluoroquinolones in the elderly: An update. Drugs and Aging, 27(3): 193209.

Sureshkumar, V., K. V. Venkateswaran and S. Jayasundar, 2004. Interaction between enrofloxacin and monensin in broiler chickens. Vet. Hum. Toxicol., 46(5): 242-245

Udayakumar, R., Sampath Kasthurirengan, Thankaraj Salammal Mariashibu, Manoharan Rajesh, Vasudevan Ramesh Anbazhagan, Sei Chang Kim, Andy Ganapathi and Chang Won Choi, 2009. Hypoglycaemic and hypolipidaemic effects of Withania somnifera root and leaf extracts on Alloxan induced diabetic rats. Int. J. Mol. Sci., 10: 23672382.

\section{How to cite this article:}

Arivuchelvan, A. and Murugesan, S. 2020. Chondroprotective Effect of Withania somnifera in Broilers Treated with High Doses of Enrofloxacin. Int.J.Curr.Microbiol.App.Sci. 9(11): 20912095. doi: https://doi.org/10.20546/ijcmas.2020.911.249 